\title{
Myocardial Infarction, CTCAE
}

National Cancer Institute

\section{Source}

National Cancer Institute. Myocardial Infarction, CT CAE. NCI Thesaurus. Code C143691.

A disorder characterized by gross necrosis of the myocardium; this is due to an interruption of blood supply to the area. 